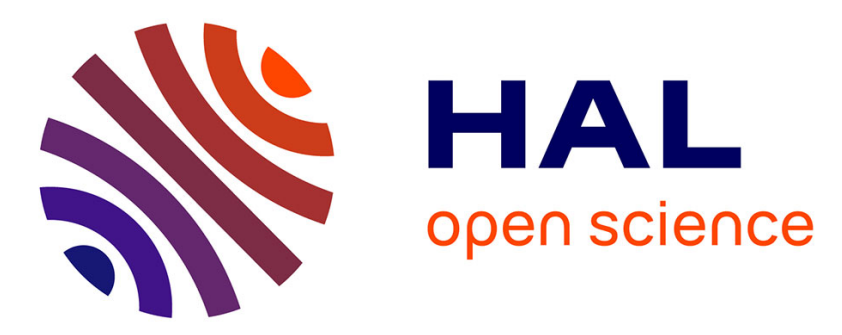

\title{
On Detectability of Moroccan Coastal Upwelling in Sea Surface Temperature Satellite Images
}

Ayoub Tamim, Khalid Minaoui, Khalid Daoudi, Abderrahman Atillah, Driss

Aboutajdine

\section{- To cite this version:}

Ayoub Tamim, Khalid Minaoui, Khalid Daoudi, Abderrahman Atillah, Driss Aboutajdine. On Detectability of Moroccan Coastal Upwelling in Sea Surface Temperature Satellite Images. 10th International Symposium on Visual Computing, Dec 2014, Las Vegas, United States. hal-01078678

\section{HAL Id: hal-01078678 \\ https: / inria.hal.science/hal-01078678}

Submitted on 29 Oct 2014

HAL is a multi-disciplinary open access archive for the deposit and dissemination of scientific research documents, whether they are published or not. The documents may come from teaching and research institutions in France or abroad, or from public or private research centers.
L'archive ouverte pluridisciplinaire HAL, est destinée au dépôt et à la diffusion de documents scientifiques de niveau recherche, publiés ou non, émanant des établissements d'enseignement et de recherche français ou étrangers, des laboratoires publics ou privés. 


\title{
On Detectability of Moroccan Coastal Upwelling in Sea Surface Temperature Satellite Images
}

\author{
Ayoub Tamim ${ }^{1}{ }^{\star}$, Khalid Minaoui ${ }^{1}$, Khalid Daoudi $^{2}$, Abderrahman Atillah $^{3}$, and Driss \\ Aboutajdine $^{1}$ \\ ${ }^{1}$ LRIT Associated Unit to the CNRST-URAC n²9, Mohammed V-Agdal University, Rabat, \\ Morocco \\ ayoubtamim@gmail.com \\ 2 INRIA Bordeaux Sud-Ouest (GEOSTAT team), Talence, France \\ ${ }^{3}$ Royal Centre for Remote Sensing (CRTS), Rabat, Morocco
}

\begin{abstract}
This work aims at automatically identify the upwelling areas in coastal ocean of Morocco using the Sea Surface Temperature (SST) satellite images. This has been done by using the fuzzy clustering technique. The proposed approach is started with the application of Gustafson-Kessel clustering algorithm in order to detect groups in each SST image with homogenous and non-overlapping temperature, resulting in a $c$-partitioned labeled image. Cluster validity indices are used to select the $c$-partition that best reproduces the shape of upwelling areas. An area opening technique is developed that is used to filter out the residuals noise and fine structures in offshore waters not belonging to the upwelling regions. The developed algorithm is applied and adjusted over a database of 70 SST images from years 2007 and 2008, covering the southern part of Moroccan atlantic coast. The system was evaluated by an oceanographer and provided acceptable results for a wide variety of oceanographic conditions.
\end{abstract}

Keywords: Upwelling, sea surface temperature, fuzzy clustering, Gustafson-Kessel clustering algorithm, validity indices, area opening.

\section{Introduction}

The Moroccan Atlantic coast, located in the central canary system, is characterized by the presence of upwelling activity almost all around the year [1]. In fact, the Moroccan coastal upwelling is evidenced at the surface by cold waters near the coast in response to the intensification of northeasterly winds and Ekman transport along the Moroccan continental shelf.

The upwelling process promotes high primary productivity by dispersing nutrients and organisms over the surface layer, favouring the blooming of phytoplankton concentration and the decreasing in sea surface temperatures in onshore direction. In particular, the thermal upwelling fronts, separating two waters masses of distinct temperatures, aggregate the organisms along their boundaries, which attract mobile predators such as fish, birds and marine mammals [2]. The thermal upwelling fronts are the boundaries

\footnotetext{
* This work is funded by the French-Moroccan research program Volubilis (MA/11/256) and the project $\mathrm{n}^{\circ}$ MPI $12 / 2010$.
} 
that all the oceanic investigators are interested in describing and identifying [3].

Infrared satellite images have demonstrated to be an effective tool for detecting the underlying upwelling structures. The proliferation and rapid expansion of infrared satellite data has allowed researchers to develop automatic methods for detecting and analyzing the upwelling fronts, in order to deal with the huge amount of satellite data daily collected and analyzed by the oceanographers. The sea surface temperature (SST) satellite images obtained from the Advanced Very High Resolution Radiometer (AVHRR) sensor aboard the NOAA satellite, are frequently used to localise the upwelling boundaries between the cold upwelling waters near the coast and warmer offshore waters [4-6]. These thermal boundaries on SST images, are usually associated to the weak or strong horizontal gradient in response to the interaction of a complex set of cold and warm waters.

In the literature several techniques and algorithms have been proposed in order to automatically detect the upwelling fronts in SST images. They are, for example, the histogram-based separation [3], where the bimodality of SST histogram is interpreted to represent two populations of water masses (cold and warm waters), and the neural networks approach [7], which classifies SST images using a feed-forward backpropagation neural network in order to find regions of homogeneous and uniform temperatures. Another class of methods deals directly with the temperature values in SST images by using the fuzzy clustering techniques $[5,6,8]$. The latter has demonstrated an effectiveness to detect the thermal boundaries in SST images, based on the fact that infrared satellite images are imprecise in nature and the associated frontal boundaries are often related to the smooth transitions between the cold and warm waters [5].

Most recently, the work reported in [6], explores the applicability of the Fuzzy $c$ means (FCM) algorithm for the detection of the main upwelling front in coastal ocean of Morocco using the SST images. The algorithm seems promising, since it accurately detect the main front with certain degree of fuzziness. However, the FCM algorithm used in the previous studies is computed with the Euclidean distance norm, and consequently it can only detect clusters with hyper-spherical shape. In contrast, the upwelling waters have strong morphological variation due to the horizontal alterations in the distribution of the physical, chemical and biological properties of the surface waters [9]. In this sense, and in order to counteract this difficulty, the current work uses the GutafsonKessel algorithm, which is an extended version of the standard fuzzy $c$-means algorithm with adaptive distance norm, for the purpose of detecting clusters with various geometrical shapes and sizes.

The outline of the paper is as follows. Section 2 describes the study area and data used throughout this paper. Section 3 describes the overview of the used algorithm. Section 4 and Section 5 present respectively the upwelling detection and upwelling segmentation steps of the proposed methodology for the automatic detection of the areas covered by upwelling waters. The experimental study and results are discussed in Section 6. Conclusions and future work are presented in Section 7. 


\section{Study area and data}

\subsection{Study area}

This study focuses on the southern part of Moroccan atlantic coast located in the central canary current system, between $27^{\circ} 60^{\prime} \mathrm{N}, 13^{\circ} 35^{\prime} \mathrm{W}$ and $20^{\circ} 0^{\prime} \mathrm{N}, 23^{\circ} 30^{\prime} \mathrm{W}$. This region experiences strong and persistent upwelling activity throughout the year [1]. The area includes the upwelling fronts which are the recently upwelled waters, colder and nearer the coast and the upwelling filaments representing the extending of upwelling fronts in offshore.

\subsection{Satellite data}

Infrared $\sim 1.0 \mathrm{~km}^{2}$ spatial pixel resolution SST images are obtained by the Royal Centre of Remote Sensing (CRTS) of Morocco from the NOAA AVHRR on polar orbiting environmental satellites NOAA-15, NOAA-16, NOAA-17, and NOAA-18. The SST images are geometrically corrected and radiometrically calibrated, so that each image is composed of $770 \times 990$ pixels with temperature information in Celsius degrees. A cloud overlay is generated using a sequence of multispectral signature threshold, tested over the AVHRR images, in order to achieve a rough segmentation of the data between non-cloudy and possibly cloudy pixels [10].

For the purpose of removing SST images with heavy cloud occlusions and artifacts over the area of interest, each SST image is kept for further analysis if it has at least $70 \%$ of valid information (no clouds or missing pixels) in the area between the coast and $200 \mathrm{~km}$ offshore [11]

In addition to daily SST images used in this study, 46 weekly synthetic images are included in our database where only the maximum pixels values in 8 daily images are retained based on the maximum value composite method [12]. The rational for choosing the weekly synthetic products is to eliminate a maximum number of pixels contaminated by clouds and reducing the large number of images daily processed [6]. In total, 70 AVHRR SST images are used to provide a coarse segmentation of upwelling areas, covering the years 2007 and 2008.

To enhance the visual detection (made by the oceanographers) of the upwelling regions in images, the SST data are linearly rescaled to 26-color scales. Fig. 1 shows two SST images selected from the 70 AVHRR data, illustrating two upwelling scenarios encountered throughout this work: SST images with a well charaterized upwelling structures in terms of clear and visible thermal front separating the upwelling waters near the coast and warmer offshore waters (Fig. 1(a)); SST images where the clouds contamination may prevent the proper segmentation of the upwelling areas (Fig. 1(b)).

\section{Overview of the proposed methodology}

The methodology implemented to detect and localize the upwelling regions in SST images is summarized in the following two steps: 


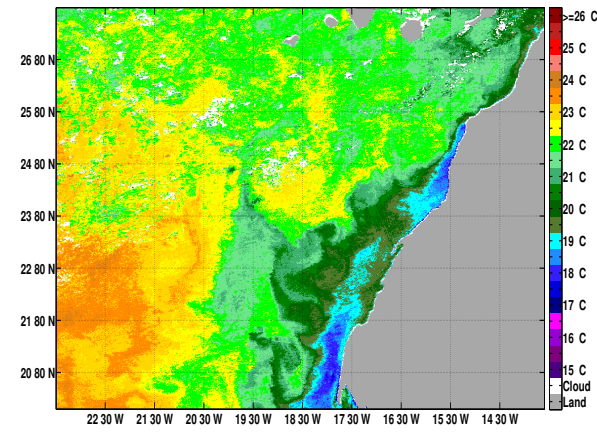

(a)

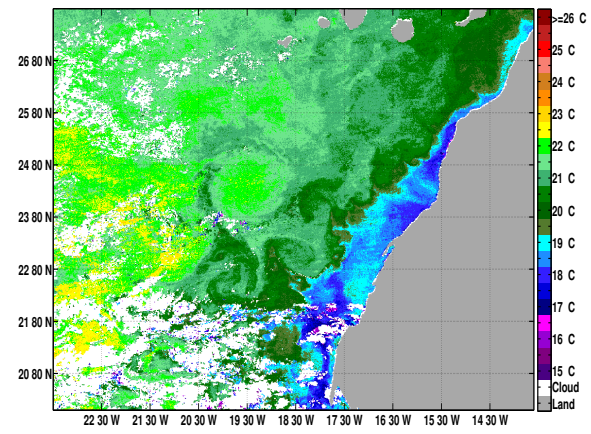

(b)

Fig. 1: SST images obtained respectively on (a) 2008-11-40 and (b) 2007-05-07, illustrating different upwelling situations.

i) Upwelling detection step, to provide a first segmentation of upwelling areas using a fuzzy clustering technique with clusters validity functions; followed by a simple thresholding stage that extracts the upwelling regions from non-upwelling regions.

ii) Upwelling segmentation step, to remove the noisy structures not belonging to the upwelling regions from a binary image result, based on the area opening algorithm.

Section 4 and Section 5 describes the two preceding steps, respectively.

\section{Upwelling detection methodology}

\subsection{Fuzzy clustering algorithm}

As a first step, we apply to each SST image a fuzzy clustering technique using the Gustafson-Kessel (GK) algorithm, to provide labeled clusters having similar statistical properties. The GK algorithm [13] is the extended version of the standard fuzzy $c$-means algorithm, that makes use of an adaptive distance norm, in order to detect clusters with different geometrical shapes and size in the dataset.

As mentioned, the upwelling waters present strong morphological variation and consequently the standard FCM algorithm, computed with the Euclidean distance norm, is not well-adapted for this application. In this sense, each SST image is converted into a feature vector $X=\left\{x_{1}, x_{2}, \ldots, x_{N}\right\}$ of $N$ pixels and the GK algorithm is applied to that vector by setting the number of clusters varying from $c_{\min }=2$ to $c_{\max }=7$, since an experimental study carried out for this work has shown that the change in the mean temperature of the main clusters is not important for more than 7 clusters.

The Gustafson-Kessel [13] algorithm is based on iterative optimization of an objective function called $c$-means functional:

$$
J(X ; U, V)=\sum_{i=1}^{c} \sum_{j=1}^{N}\left(\mu_{i j}\right)^{m}|| x_{j}-v_{i} \|_{A_{i}}^{2},
$$


where $N$ is the total number of pixels in a given SST image, $V=\left\{v_{1}, v_{2}, \ldots, v_{c}\right\}$ is a vector of cluster prototypes and $U=\left[u_{i j}\right]_{c \times N}$ is a fuzzy partition matrix composed of the membership grade of object $x_{j}$ to each cluster $i$. The weighting exponent $m$, which vary from $[1, \infty)$, is a constant that influences the membership values, determining the degree of fuzziness of the cluster partition. Values of $m \rightarrow \infty$, lead to entirely fuzzy partitions while as $m \rightarrow 1.0$, the fussy partitions reduces to the crisp or hard partitions. In our implementation, we set this parameter to 2 .

In GK algorithm, each cluster has its own norm-inducing matrix $A_{i}$, which yields the following inner-product norm:

$$
\left\|x_{j}-v_{i}\right\|_{A_{i}}^{2}=\left(x_{j}-v_{i}\right)^{T} A_{i}\left(x_{j}-v_{i}\right)
$$

The matrices $A_{i}$ are used as optimization variables in the objective function (Eq. 1), thus allowing each cluster to adapt the distance norm to the local topological structure of the data.

\subsection{Optimal number of clusters}

Given the collection of fuzzy $c$-partitions labeled images, one needs to determine the optimal number of clusters, $c^{*}$-partition, which correspond to the real groups in data. To this end, the clusters validity functions are widely used for this purpose as a mechanism to quantitatively validate the best description of the data structure and then extract the optimal number of clusters.

A number of validity indices for fuzzy clustering exist in the literature [14]. In particular, the Xie-Beni $\left(V_{X B}\right)$ [15] and Fukuyam-Sugeno $\left(V_{F S}\right)$ [16] functions have been frequently used due to their computational efficiency and effectiveness for estimating the good number of clusters [17]. Consequently, these two indices are used in the current work to assess the quality of the obtained $c$-partitions. A small value of $V_{X B}$ and $V_{F S}$ means a fuzzy $c$-partition with compact and well-separated clusters. Hence, the best fuzzy $c$-partition is obtained by taking the global minimum of $V_{X B}$ and $V_{F S}$ with respect to $c=2,3, \ldots, c_{\max }$.

The results of the two validity indices $\left(V_{X B}\right.$ and $\left.V_{F S}\right)$, applied to the original SST images in Fig. 1 using the GK algorithm with number of cluster varying from $c_{\min }=2$ to $c_{\max }=7$, are shown in Fig. 2 . As shown, the absolute minimum of the two curves is reached by the clusters number $c^{*}=2$, which is consistent with the suitable number of cluster to reproduce the areas covered by upwelling waters for SST images in Fig. 1, and also for all the images analyzed in this study.

Once the labeled image is generated using the Gustafson-Kessel algorithm with the optimal number of clusters, $c^{*}=2$, the upwelling area is defined and flagged as the clusters with the lowest mean value, based on the fact that the upwelling region is characterized by a cold temperature waters compared to the offshore warmer waters [6]. Fig. 3 shows the binary image result after the upwelling detection methodology. The contiguous white region on the binary images in Fig. 3 corresponds to upwelling area, whereas the black pixels correspond to offshore waters non affected by the coastal upwelling. 


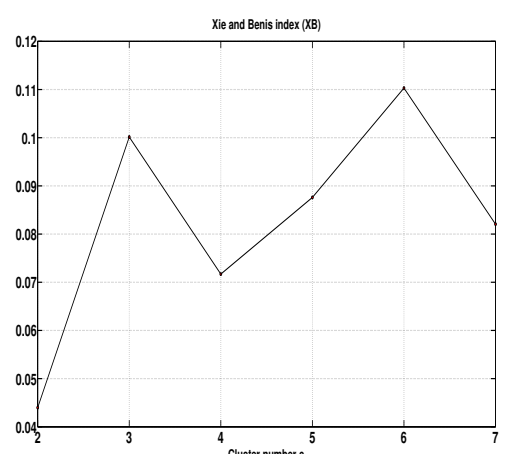

(a)

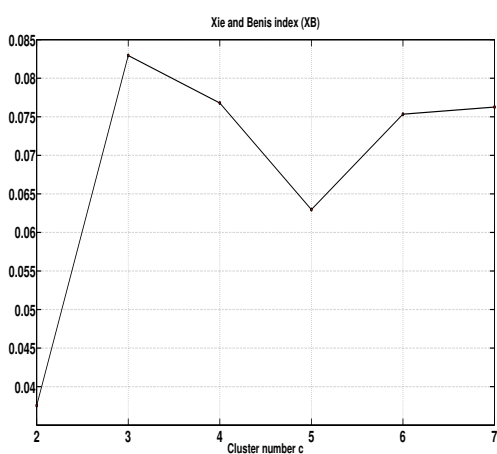

(c)

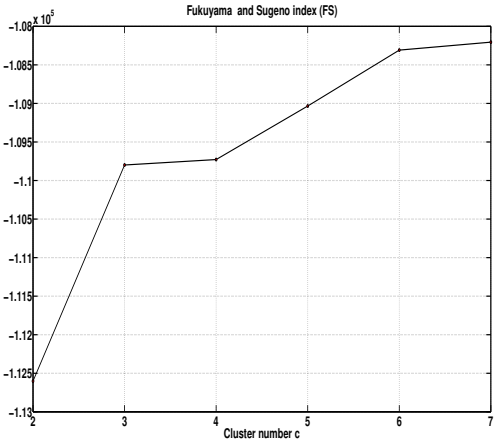

(b)

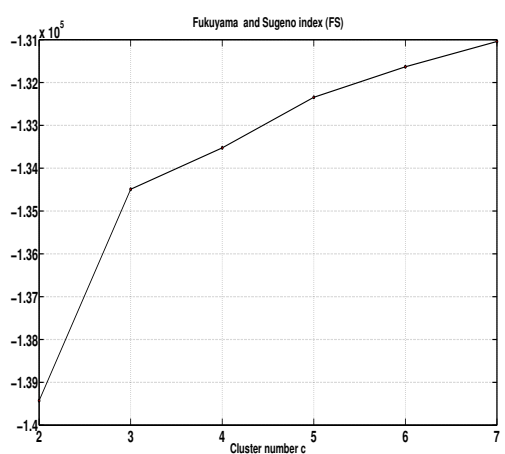

(d)

Fig. 2: Results of the validity indices with GK algorithm using respectively the $V_{X B}$ and $V_{F S}$ indices to the SST images in Fig. 1(a) ((a) and (b)) and Fig. 1(b) ((c) and (d)).

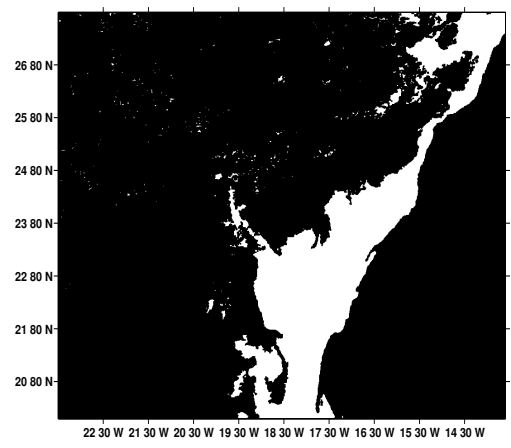

(a)

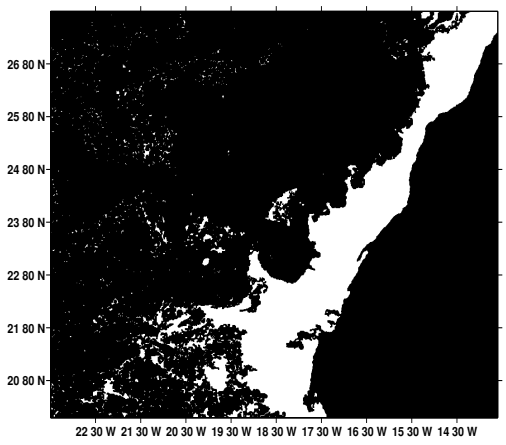

(b)

Fig. 3: Binary image result after the upwelling detection step for respectively the two SST images in Fig. 1. 


\section{Upwelling segmentation methodology}

Unfortunately, the binary images results in Fig. 3 include upwelling area plus additional noise structures in offshore waters not belonging to the upwelling region, mainly due to the presence of fine structures and cloud contamination pixels in offshore that are not identified by the cloud detection algorithm used in this study [10]. Therefore, a segmentation procedure is required consisting on the application of the area opening algorithm to remove pixels and contiguous regions faraway from the coast, not belonging to the upwelling waters.

The area opening is a transformations using a structuring element which locally adapts its shape to the image structures, and therefore filtering out small regions (lower than a given threshold) without damaging the remaining structures in the images [18]. This method seems to be relevant to our problem of removing the cold small regions in offshore waters (the white pixels on the left side of images in Fig. 3) without deteriorating the upwelling area.

An evaluation has been carried out for this study using the 70 SST AVHRR images, in order to select a single threshold value that remove the noisy structures from the images. After an extensive evaluation, good results are obtained by the threshold value of 200 pixels. So, each regions less than 200 pixels, will be discarded from the given image. Fig. 4 shows the results of the area opening algorithm applied to the binary images of Fig. 3 with the thresholding value fixed at 200 pixels. As we can see, all the remaining pixels in offshore waters have been removed and separated from the upwelling area. Fig. 5 shows the final segmentation results achieved by our proposed methodology, where the upwelling area is automatically contoured with black bold color.

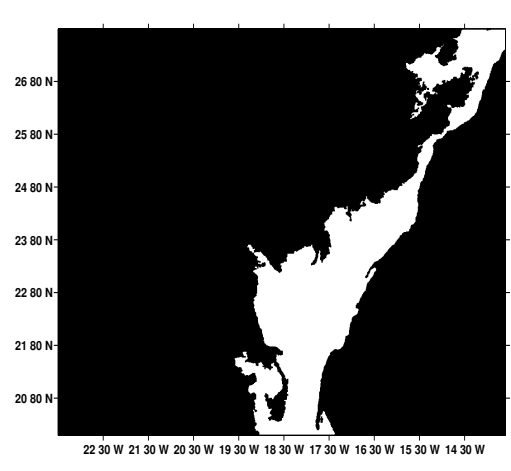

(a)

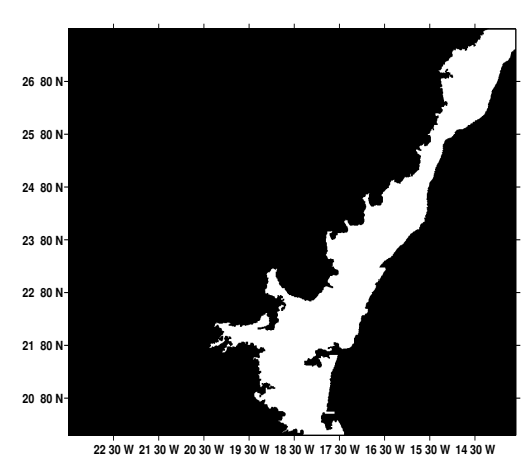

(b)

Fig. 4: Retrieved upwelling areas using the area opening algorithm of the two binary images results of Fig. 3. 


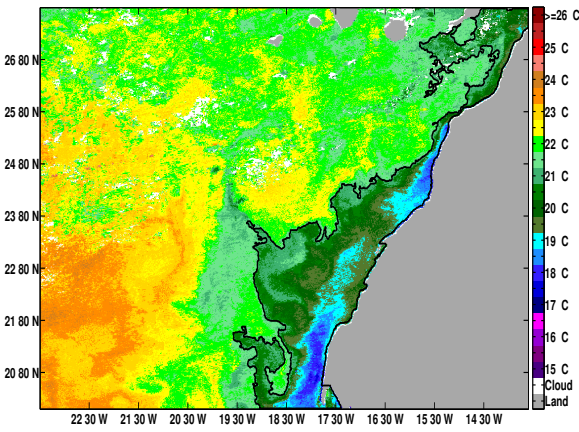

(a)

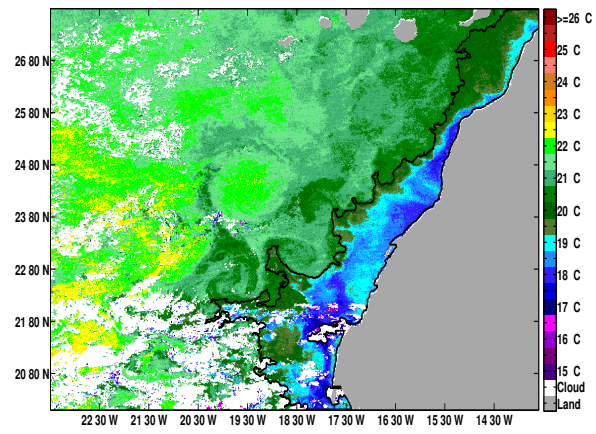

(b)

Fig. 5: Upwelling region automatically contoured using the proposed methodology to the SST images in Fig. 1.

\section{Experimental results and discussion}

To evaluate the performance of the proposed methodology of detecting the Moroccan coastal upwelling, it is validated using the visual inspection made by the oceanographer based on the scientific and technical knowledge of the Moroccan atlantic coast.

The in-situ measurement, e.g., buoys data, can give us a sounder support to the results obtained by the proposed algorithm. However, these measures are very complex to establish and would be extremely challenging for a use in coastal zone context, because of lack of these measures for all the satellite data and for every specific region in the study area. In this sense, it is a well-known fact that validation in the case of ocean data is often and truly performed by assessing the results by professional oceanographers $[5$, $6,8]$, which has been done in the case of this work.

The oceanographic interpretation is applied to check if the obtained results correctly identify the upwelling area without over-detection or missing-detection of the structures. For this purpose, four grades are used: "Bad", "Poor", "Good" and "Excellent" in order to achieve the oceanographic evaluation. The grade "Bad" is attributed when the upwelling area is not well-segmented, and "Excellent" is marked when the upwelling region is correctly delimitated. The results of the oceanographer interpretation for our segmentation methodology of 70 SST images, are presented in Fig. 6. As we can see, the Fig. 6 shows that the grades "Bad" and "Poor" reached respectively the values $2 \%$ and $6 \%$, resulting in a total of $8 \%$ of 70 SST images. In contrast, the values of $58 \%$ and $34 \%$ are reached respectively by the grades "Good" and "Excellent", making a total of $92 \%$. The marks attributed to the images in Fig. 5(a) and Fig. 5(b) are respectively "Excellent" and "Good".

After this evaluation of the segmentation methodology, over this representative database, we can conclude that the proposed algorithm has provided satisfactory results. 


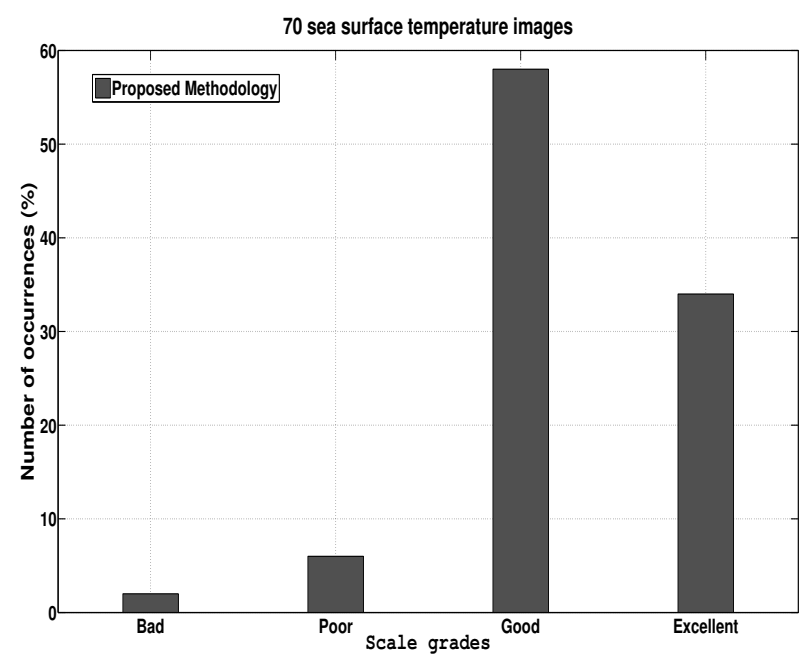

Fig. 6: Qualitative interpretation made by the oceanographer of the 70 SST images for the proposed approach using the Gustafson-Kessel algorithm.

\section{Conclusions and future work}

In this paper we have presented a classification methodology to deal with the problem of precise segmentation of Moroccan coastal upwelling using the SST images. To accomplish this task the algorithm is composed by two phases. The first one with the goal to detect the upwelling area by the application of Gustafson-Kessel clustering algorithm and cluster validity indices, that generate labeled image with homogeneous groups of pixels and no-overlapping classes, followed by a thresholding stage that identify the lowest mean cluster as the upwelling region. The second phase achieves the segmentation procedure to eliminate isolated components, in offshore waters, not belonging to the upwelling structure, based on the area opening algorithm.

For comparison purposes, the algorithm is applied to 70 AVHRR SST images on board the NOAA satellite and evaluated using the scientific knowledge of the Moroccan costal upwelling provided by the oceanographer. The proposed algorithm has demonstrated a great performance for a majority of images used in this study, and it is able to objectively segment the upwelling area in automatic manner and without the intervention of the human operators. Our future work will consist in using this segmentation to other satellite data products, such as the remotely sensed sea-surface chlorophyll field, to give us a sounder support to the results already obtained. 


\section{References}

1. Atillah, A., Orbi, A., Hilmi, K., Mangin, A.: Produits opérationnels d'océanographie spatiale pour le suivi et l'analyse du phénomène d'upwelling marocain. Geo Observateur 14 (2005) 49-62

2. Bakun, A.: Fronts and eddies as key structures in the habitat of marine fish larvae : Opportunity, adaptative response and competitive advantage. Scientia Marina 70 (2006) 105-122

3. Cayula, J.F., Cornillon, P.: Edge detection algorithm for SST images. Journal of Atmospheric and Oceanic Techology 9 (1992) 67-80

4. Holyer, R.J., Peckinpaugh, S.H.: Edge detection applied to satellite imagery of the oceans. IEEE Transactions on Geoscience and Remote Sensing 27 (1989) 46 - 56

5. Nascimento, S., Franco, P., Sousa, F., Dias, J., Neves, F.: Automated computational delimitation of SST upwelling areas using fuzzy clustering. Computers and Geosciences 43 (2012) 207-216

6. Tamim, A., Minaoui, K., Daoudi, K., Yahia, H., Atillah, A., Smiej, M.F., Aboutajdine, D.: A simple and efficient approach for coarse segmentation of Moroccan coastal upwelling. In: Signal Processing Conference (EUSIPCO), 2013 Proceedings of the 21st European. (2013) $1-5$

7. Chaudhari, S., Balasubramanian, R., Gangopadhyay, A.: Upwelling detection in AVHRR sea surface temperature (SST) images using neural-network framework. IEEE International Geoscience Remote Sensing Symposium II (2008) 926-929

8. Sousa, F.M., Nascimento, S., Casimiro, H., Boutov, D.: Identification of upwelling areas on sea surface temperature images using fuzzy clustering. Remote Sensing of Environment 112 (2008) $2817-2823$

9. Marcello, J., Marques, F., Eugenio, F.: Automatic tool for the precise detection of upwelling and filaments in remote sensing imagery. IEEE Transactions on Geoscience and Remote Sensing 43 (2005) 1605-1616

10. Stowe, L.L., Davis, P.A., McClain, E.P.: Scientific basis and initial evaluation of the clavr-1 global clear/cloud classification algorithm for the advanced very high resolution radiometer. Journal of Atmospheric and Oceanic Technology 16 (1999) 656

11. Nieto, K., Demarcq, H., McClatchie, S.: Mesoscale frontal structures in the canary upwelling system: New front and filament detection algorithms applied to spatial and temporal patterns. Remote Sensing of Environment 123 (2005) 49-62

12. Holben, B.N.: Characteristics of maximum-value composite images from temporal AVHRR data. International Journal of Remote Sensing 7 (1986) 1417-1434

13. Gustafson, D., Kessel, W.: Fuzzy clustering with a fuzzy covariance matrix. In: Decision and Control including the 17th Symposium on Adaptive Processes, 1978 IEEE Conference on. (1978) 761-766

14. Wang, W., Zhang, Y.: On fuzzy cluster validity indices. Fuzzy Sets and Systems 158 (2007) $2095-2117$

15. Xie, X.L., Beni, G.: A validity measure for fuzzy clustering. Pattern Analysis and Machine Intelligence, IEEE Transactions on 13 (1991) 841-847

16. Fukuyama, Y., Sugeno, M.: A new method of choosing the number of clusters for the fuzzy c-means method. In: Proceeding of fifth Fuzzy System Symp. (1989) 247-250

17. Nascimento, S., Franco, P.: Segmentation of upwelling regions in sea surface temperature images via unsupervised fuzzy clustering. In Corchado, E., Yin, H., eds.: Intelligent Data Engineering and Automated Learning - IDEAL 2009. Volume 5788 of Lecture Notes in Computer Science. Springer Berlin Heidelberg (2009) 543-553

18. Vincent, L.: Morphological area openings and closings for grey-scale images. In: Shape in Picture. Volume 126. Springer Berlin Heidelberg (1994) 197-208 